\title{
Scaffolded Self-Explanation with Visual Representations Promotes Efficient Learning in Early Algebra
}

\author{
Tomohiro Nagashima (tnagashi@cs.cmu.edu) \\ Carnegie Mellon University, 5000 Forbes Ave., Pittsburgh, PA 15213, USA \\ Anna N. Bartel (anbartel@wisc.edu) \\ University of Wisconsin, Madison, 1202 West Johnson Street, Madison, WI 53706-1611, USA \\ Stephanie Tseng (stseng2@andrew.cmu.edu) \\ Carnegie Mellon University, 5000 Forbes Ave., Pittsburgh, PA 15213, USA \\ Nicholas A. Vest (navest@wisc.edu) \\ University of Wisconsin, Madison, 1202 West Johnson Street, Madison, WI 53706-1611, USA \\ Elena M. Silla (esilla@wisc.edu) \\ University of Wisconsin, Madison, 1202 West Johnson Street, Madison, WI 53706-1611, USA \\ Martha W. Alibali (mwalibali@wisc.edu) \\ University of Wisconsin, Madison, 1202 West Johnson Street, Madison, WI 53706-1611, USA \\ Vincent Aleven (aleven@cs.cmu.edu) \\ Carnegie Mellon University, 5000 Forbes Ave., Pittsburgh, PA 15213, USA
}

\begin{abstract}
Although visual representations are generally beneficial for learners, past research also suggests that often only a subset of learners benefits from visual representations. In this work, we designed and evaluated anticipatory diagrammatic selfexplanation, a novel form of instructional scaffolding in which visual representations are used to guide learners' inference generation as they solve algebra problems in an Intelligent Tutoring System. We conducted a classroom experiment with 84 students in grades 5-8 in the US to investigate the effectiveness of anticipatory diagrammatic self-explanation on algebra performance and learning. The results show that anticipatory diagrammatic self-explanation benefits learners on problem-solving performance and the acquisition of formal problem-solving strategies. These effects mostly did not depend on students' prior knowledge. We analyze and discuss how performance with the visual representation may have influenced the enhanced problem-solving performance.
\end{abstract}

Keywords: visual representations; diagrams; self-explanation; learning; tape diagrams; middle-school algebra

\section{Introduction}

\section{Visual Representations as Instructional Scaffolding}

Instructional scaffolding is any form of external assistance that helps learners achieve their learning goals (Delen et al., 2014). Instructional scaffolding can take a variety of forms, including chunking, explanation, instructional cues, and worked examples, to help learners engage in the target task in a less cognitively-demanding way (e.g., Barbieri et al., 2019). One form of instructional scaffolding is visual representations, such as diagrams. Visual representations are frequently used across domains and have generally been shown to enhance performance and learning (Rau, 2017).

Despite the widespread use and effectiveness of visual representations, many studies also report that visual representations are not universally beneficial. In particular, learners with low prior knowledge and younger learners do not consistently benefit from learning with visual representations (e.g., Booth \& Koedinger, 2012), and in some cases, visual representations are actually detrimental (e.g., Magner et al., 2014). Therefore, research is needed to understand variations in the benefits of visual representations and the mechanisms through which visuals help or fail to help learning and performance.

Middle-school algebra is one domain in which visual representations are regularly used (Murata, 2008). Researchers have investigated the effectiveness of a specific type of visual representation, called "tape diagrams" alongside algebra problems as an instructional aid for problem solving (Bartel et al., 2021; Booth \& Koedinger, 2012; Chu et al., 2017). Tape diagrams depict relations between different quantities in an equation or mathematical situation (Figure 1; Murata, 2008). Prior studies have shown that adding tape diagrams to symbolic equations or word problems can enhance middle-school students' problemsolving performance and help avoid conceptual errors (Booth \& Koedinger, 2012; Chu et al., 2017). However, as previously discussed in regard to using visual representations as scaffolding, the effects of tape diagrams are not consistent across students with varying levels of prior knowledge in the target domain. Specifically, students with lower prior knowledge or in lower grade levels do not tend to benefit 
from tape diagrams (Booth \& Koedinger, 2012) and they have difficulty understanding the information conveyed through the diagram (Chu et al., 2017).

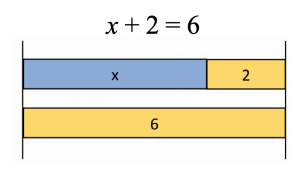

Figure 1: An example of tape diagrams. The tapes together represent the equation, $x+2=6$.

\section{Learning with Visual Representations through Integrated Scaffolding Support}

When using visual representations as a form of instructional scaffold, one must design the scaffolding support so that learners can engage with the visual representations in a pedagogically meaningful way (Davenport et al., 2008; Nagashima, Yang et al., 2020). Merely adding visuals to the target content as an additional representation does not necessarily encourage student engagement. This is particularly true among learners with low prior knowledge, who may need extra instructional support to connect multiple representations (Booth \& Koedinger, 2012; Davenport et al., 2008). Therefore, in order to support learners with varied levels of prior knowledge, it is essential to design additional instruction or activities that appropriately scaffold learning through interactive and integrative use of visual representations (e.g., Nagashima, Bartel et al., 2020).

Prior studies have integrated various types of interactive support for the use of visual representations. In particular, studies have tested the effect of using self-explanation with visual representations (Ainsworth \& Loizou, 2003; Aleven \& Koedinger, 2002; Butcher \& Aleven, 2013; Rau et al., 2015) and, specifically, with tape diagrams (Nagashima, Bartel et al., 2020). Self-explanation is an established learning strategy in which learners make sense of new information by generating an explanation that connects the new information to their existing knowledge (Chi et al., 1989). Self-explaining target content with the help of visual representations provides an opportunity for learners to explicitly make connections between the target representation (e.g., mathematical symbols) and the visual representation. Indeed, a prior study found that integrating tape diagrams into self-explanation enhances learning (Nagashima, Bartel et al., 2020). However, studies rarely explore whether self-explanation can promote efficient learning (e.g., "do learners spend less time if they self-explain?") (Bisra et al., 2018; but see Aleven \& Koedinger, 2002) and studies rarely seek to identify the mechanisms through which self-explanation with visual representations helps efficient performance and learning. As designing an appropriately-scaffolded self-explanation activity is key for its benefits (Nagashima, Yang et al., 2020), it is important to design and examine whether and how selfexplanation as scaffolding support contributes to instructional effectiveness and efficiency when learning with visual representations.

\section{Anticipatory Diagrammatic Self-Explanation}

We designed a novel form of self-explanation support for learning with visual representations called anticipatory diagrammatic self-explanation (Nagashima et al., 2021). Anticipatory diagrammatic self-explanation integrates visual representations, contrasting cases (Schwartz et al., 2011), and anticipatory self-explanation (Renkl, 1997) to support both learning and performance in a highly scaffolded way. In anticipatory diagrammatic self-explanation, learners infer future problem-solving steps, in contrast to the more common form of self-explanation during problem solving, in which learners provide an explanation for the step that they have already completed or seen (e.g., Rau et al., 2015).

We implemented anticipatory diagrammatic selfexplanation using tape diagrams in the context of algebra equation solving in an Intelligent Tutoring System (ITS). Figures 2 and 3 show the step-by-step interaction that takes place in anticipatory diagrammatic self-explanation. Learners first engage in a diagram selection task in which they infer, or explain, the next strategic problem-solving step. Then, they enter the corresponding problem-solving step expressed in symbols on the right side of the screen. The diagrammatic and symbolic representations are tightly linked; the ITS asks learners to try again if they type in a symbolic step that does not follow the diagram selected, even if it is algebraically correct. The ITS also provides hints and feedback messages to support individual practices (Figure 3).

Anticipatory diagrammatic self-explanation could be effective as instructional scaffolding and potentially bring benefits to learners with different levels of knowledge. It may support the acquisition of knowledge of strategic problem solving (i.e., "which next step is correct and strategic?") by having learners engage with the representations for the next step before actually doing that step with the target representation. In anticipatory diagrammatic selfexplanation, learners are asked to select a correct-andstrategic next step in the form of a tape diagram (Figure 2). For learners with low prior knowledge who may not know how to solve the equation, this scaffolding may guide their understanding of what is correct and strategic to do, before being asked to solve the equation in mathematical symbols. For those with high prior knowledge, the diagram steps would introduce a new way of thinking about processes they already know - how to solve equations - which may further strengthen their knowledge about correct and strategic problem solving. These processes could lead to efficient problem solving (i.e., solving problems with a low error rate, little use of hints, and within less time).

These expected benefits may be accompanied by other potential benefits introduced by visual representations and contrasting cases. For example, engaging with a visual representation in the form of contrasting cases may help students understand algebra concepts, such as isolating the variable (Nagashima, Bartel et al., 2020; Schwartz et al., 2011). Also, problem solving within an ITS may help students acquire problem-solving skills, as shown in past work with ITSs (e.g., Long \& Aleven, 2013). 


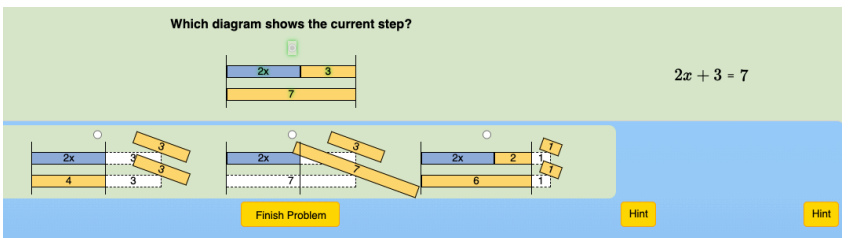

Figure 2: Learners first select a correct-and-strategic diagram option for the next problem-solving step. They receive feedback on the correctness of the selected option.

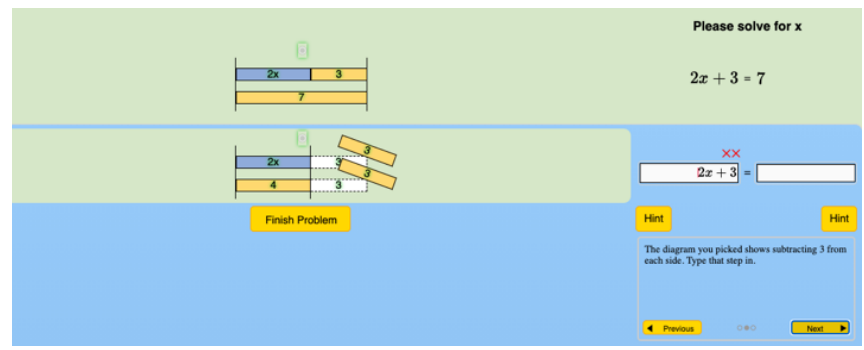

Figure 3: After selecting a correct diagrammatic step, learners are asked to solve the step with mathematical symbols. The ITS gives feedback and hints. Here the feedback says, "The diagram you picked shows subtracting 3 from each side. Type that step in."

In the present study, we investigate whether and how anticipatory diagrammatic self-explanation enhances students' performance, learning, and understanding of the formal problem-solving strategy. We also consider whether anticipatory diagrammatic self-explanation is differentially effective for learners with different levels of prior knowledge.

\section{Method}

\section{Participants}

We conducted a randomized controlled classroom experiment at a single private school in the US. Participants included 305 th graders, 17 6th graders, 23 7th graders, and 21 8th graders (total $n=91$ ). ${ }^{1}$ These students were taught by two teachers across seven class sections. We conducted the experiment in October 2020 when the school was operating under a hybrid teaching mode due to the COVID-19 pandemic. In this hybrid mode, the majority of students $(n=$ 89) attended the study in-person while two students attended remotely. Both participating teachers noted that students' prior exposure to tape diagrams was minimal; some students had seen tape diagrams in learning materials, but the instruction had never focused specifically on tape diagrams.

\footnotetext{
${ }^{1}$ Data from the $6^{\text {th }}$ and $7^{\text {th }}$ graders were also included in Nagashima et al. (2021), which reports a few overlapping results but with different research questions and types of analyses.
}

\section{Materials}

Intelligent Tutoring System for Equation Solving We developed two versions of the ITS with anticipatory diagrammatic self-explanation (described above) and another with no anticipatory diagrammatic self-explanation support, based on a prior ITS built by Long and Aleven (2014). The only difference between the versions in the study was whether or not the ITS provided the diagrammatic selfexplanation support. Other features, including step-level hints, correctness feedback, and feedback messages, were consistent across the two versions. We implemented 41 equation problems that varied in difficulty level in both ITS versions, including one-step equations $(x+\mathrm{a}=\mathrm{b})$, two-step equations $(\mathrm{a} x+\mathrm{b}=\mathrm{c})$, and equations with variables on both sides $(\mathrm{a} x+\mathrm{b}=\mathrm{c} x$ and $\mathrm{a} x+\mathrm{b}=\mathrm{c} x+\mathrm{d})$, Per teachers' reports, participating students included those who had experienced solving all of these equation types and those who had only been exposed to one-step equations. The same problem set was assigned for all participants.

Pretest and Posttest We developed a web-based pretest and posttest to assess students' conceptual knowledge (or knowledge of underlying concepts in a domain) and procedural knowledge (or problem-solving skills) of basic algebra (Crooks \& Alibali, 2014), drawn from our previous work (Nagashima, Bartel et al., 2020). The conceptual knowledge items consisted of eight multiple-choice questions and one open-ended question. They assessed a range of conceptual knowledge constructs, including inverse operations, isolating variables, and keeping both sides of an equation equal. We also included four problem-solving items (e.g., "solve for $x$ : $3 x+2=8$ "), including two items that were similar to those in the ITS and two transfer items involving negative numbers. (The problems in the ITS used positive numbers only.) None of these items included tape diagrams. We developed two isomorphic versions of the test that varied only with respect to the specific numbers used in the items; participants received one form as pretest and the other as posttest, with versions counterbalanced across subjects.

Procedure The study took place during two regular mathematics class periods, in which most of the students and the teacher were present "live" in the actual classroom, and in which experimenters and remote learners joined through a video conferencing system. Students in each class were randomly assigned to either the Diagram condition or the NoDiagram condition. In the Diagram condition, students used the ITS with anticipatory diagrammatic self-explanation. Students in the No-Diagram condition used the ITS without the self-explanation support.

On the first day, students first worked on the pretest for 15 minutes. Then the teacher or the experimenter showed students in both conditions a five-minute video describing 
how to use the ITSs and what tape diagrams represent for both conditions. Next, students practiced equation solving using their randomly-assigned ITS version for about 15 minutes. On the second day, students started the class by solving equation problems in the assigned ITS for approximately 15 minutes. After working with the ITS, students took the web-based posttest for 15 minutes. Students were given access to both ITS versions a week after the study. Figure 4 illustrates the study procedure.

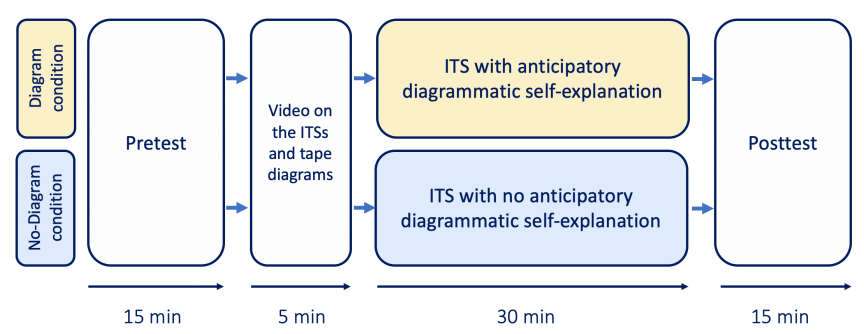

Figure 4: Study procedure. The difference between the conditions (Diagram vs No-Diagram) was whether students used the ITS with anticipatory diagrammatic selfexplanation support or the ITS without the support.

\section{Results}

Of the 91 participants, we excluded five students who did not complete the posttest and two other students who were at ceiling at the pretest. Therefore, the following analyses focus on 84 students ( 265 th graders, 16 6th graders, 237 th graders, and 21th 8 graders), of whom 41 were in the Diagram condition and 43 were in the No-Diagram condition. Two researchers evaluated all answers for the open-ended items on the pretest and posttest (840 student answers) and achieved high inter-rater reliability (Cohen's kappa $=.81$ ).

\section{Effects on Learning}

Table 1 shows pretest and posttest scores on the conceptual knowledge (CK, max: 9) and procedural knowledge (PK, max: 4) items. One-way repeated measures ANOVAs showed a significant pretest-posttest gain across conditions on the procedural knowledge items $(F(1,83)=12.88, p<.01)$ and positive but non-significant pretest-posttest gain for the conceptual knowledge items $(F(1,83)=2.86, p=.09)$. To test the effect of the intervention and its interaction with learners' prior knowledge, we conducted two separate linear regressions, one with conceptual knowledge posttest scores and one with procedural knowledge posttest scores as dependent variables. In both models, condition (Diagram or No-Diagram), prior knowledge pretest score (combined CK and PK scores), and the interaction between the two served as predictors. Additionally, the number of problems solved in the ITS and grade level were included as covariates. Grade level was treated as a continuous variable, with 5 th, 6 th, 7 th, and 8th grade coded as $-1.5,-.5, .5,1.5$, respectively. In both models, there was no significant main effect of condition $(\mathrm{CK}: \beta=-0.20, t(78)=-0.28, p=.78, \mathrm{PK}: \beta=-0.28, t(78)=$
$-0.60, p=.55)$ and no significant interaction of condition and pretest scores (CK: $\beta=.09, t(78)=.76, p=.45$, PK: $\beta=.03$, $t(79)=.39, p=.69)$.

Table 1: Pretest and posttest scores and standard deviations (in parentheses)

\begin{tabular}{ccccc}
\hline \multirow{2}{*}{ Condition } & \multicolumn{2}{c}{ Pretest } & \multicolumn{2}{c}{ Posttest } \\
\cline { 2 - 5 } & CK & PK & CK & PK \\
\hline \multirow{2}{*}{ Diagram } & 3.63 & 1.15 & 4.10 & 1.56 \\
No- & $(1.93)$ & $(1.35)$ & $(2.37)$ & $(1.52)$ \\
Diagram & 4.30 & 1.63 & 4.47 & 2.19 \\
& $(2.29)$ & $(1.57)$ & $(2.53)$ & $(1.82)$ \\
\hline
\end{tabular}

Strategy Coding To examine whether engaging with anticipatory diagrammatic self-explanation influenced students' use of formal problem-solving strategies, for the procedural items, we coded for whether students used the formal algebraic strategy in solving equations, independent of the correctness coding, following the coding scheme used in $\mathrm{Chu}$ et al. (2017). If an answer uses algebraic manipulations to reach the solution, it was coded as "Algebra" strategy. Otherwise, we coded it as "NonAlgebra" strategy, which included informal strategies such as substituting different values in the equation and providing an answer without showing any intermediate steps. Two researchers coded all 672 student answers (Cohen's kappa $=$ $.64)$.

There was no difference between the conditions in the number of students who used the Algebra strategy at least once on the procedural items at both pretest (Diagram: 12 out of 41, No-Diagram: 20 out of $43, \chi 2[1, n=84]=2.65, p=$ .10 ) and posttest (Diagram: 16 out of 41, No-Diagram: 22 out of 43, $\chi 2[1, n=84]=1.25, p=.26)$. However, for the two transfer items, we found a significant increase in the number of students using the Algebra strategy from pretest to posttest for the Diagram condition (from 7 to $16, p=.01$ ), but not for the No-Diagram condition (from 16 to $18, p=.72$ : Figure 5 ).
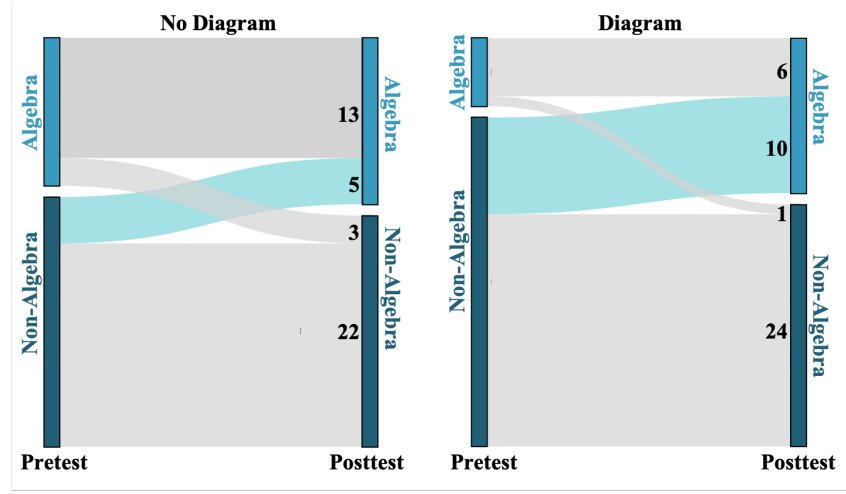

Figure 5: The change, by condition (left: No-Diagram; right: Diagram), from pretest to posttest, in the number of students who used the Algebra strategy 


\section{Effects on Performance in the ITS}

To investigate students' performance in the ITS, we analyzed $\log$ data collected by the ITS. Specifically, we explored the total number of problems solved, the average number of incorrect attempts at each problem-solving step, the average number of hints requested at each step, and the time spent on each step (Table 2), which are process measures typically used in the literature (e.g., Long \& Aleven, 2013). We only compare the process measures on the symbolic steps, excluding the transactions for the diagrammatic steps, to make fair comparisons between the conditions.

Table 2: The means and standard deviations (in parentheses) of the process measures

\begin{tabular}{ccccc}
\hline Condition & $\begin{array}{c}\text { Number } \\
\text { of } \\
\text { problems } \\
\text { solved }\end{array}$ & $\begin{array}{c}\text { Average } \\
\text { number of } \\
\text { incorrect } \\
\text { attempts } \\
\text { per step }\end{array}$ & $\begin{array}{c}\text { Average } \\
\text { number of } \\
\text { hints } \\
\text { requested } \\
\text { per step }\end{array}$ & $\begin{array}{c}\text { Average } \\
\text { time } \\
\text { spent per } \\
\text { step }\end{array}$ \\
\hline Diagram & 14.22 & 1.07 & 0.31 & 18.42 \\
No- & $(8.47)$ & $(2.03)$ & $(0.52)$ & $(13.02)$ \\
Diagram & $(13.94)$ & 1.09 & 0.56 & 21.30 \\
& & $(1.57)$ & $(0.72)$ & $(17.15)$
\end{tabular}

To examine whether learners in the Diagram condition showed efficient learning, we ran four separate linear regressions with each of the process measures as a dependent variable. In all four models, condition, pretest score, and their interaction were included as independent variables. Additionally, grade level was included as a covariate. Also, we added the number of problems solved as a covariate to three of the four models (the ones in which it was not the dependent variable) because the number of problems solved was strongly/moderately correlated with each of the three other dependent variables.

First, we found a main effect of pretest scores on the number of problems solved, $\beta=3.04, t(79)=7.49, p<.01$, indicating that as prior knowledge increases, students solved more problems in the ITS. This increase was steeper for students in the No-Diagram condition than the Diagram condition, $\beta=-1.24, t(79)=-2.12, p=.04$ (Figure 6). We then tested simple main effects of condition at one standard deviation below the mean for combined pretest scores and one standard deviation above the mean for combined pretest scores (see dotted vertical lines in Figure 6). Results showed that among those who scored above average on the pretest, learners in the No-Diagram condition solved significantly more problems than those in the Diagram condition. $\beta=3.53$, $t(79)=2.69, p<.01$. However, there was no difference in the number of problems solved between conditions for learners who scored below average on the pretest, $\beta=-0.40, t(79)=$ $0.31, p=.76$.

Regarding hint use and average time spent per step, we found a significant main effect of condition (hint use: $\beta=$ $0.71, t(78)=-3.08, p<.01$; time per step: $\beta=-12.18, t(78)=$ $-2.89, p<.01)$ but no significant interactions between condition and pretest score. There were no significant main nor interaction effects on the average number of incorrect attempts made per step. These results indicate that anticipatory diagrammatic self-explanation helped learners spend less time and request fewer hints on symbolic steps than learners with no self-explanation support, but it did not help them make fewer errors on symbolic steps.

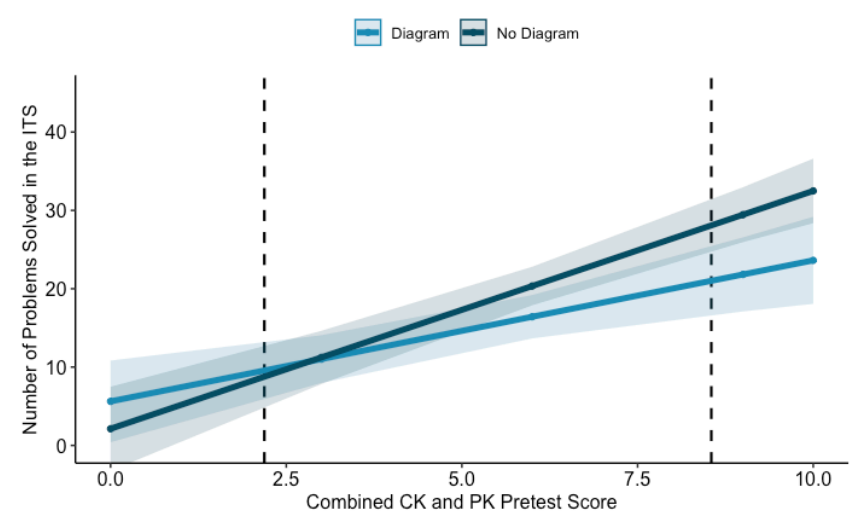

Figure 6: An interaction between condition and pretest score on the number of problems solved. The slope is steeper for the No-Diagram condition than the Diagram condition. The two dotes lines indicate our two tests of simple main effects; one standard deviation below and one standard above the mean for combined pretest score.

To uncover how the anticipatory diagrammatic selfexplanation scaffolded student performance, we examined relations between performance on the diagrammatic steps and the symbolic steps using ITS log data from the participants in the Diagram condition $(n=41)$. We tested if any of the performance measures for diagrammatic steps predicted learners' performance on symbolic steps. We ran three additional linear regressions with the same set of predictors of primary interest: pretest scores, the average number of incorrect attempts for each diagrammatic step, and the average time spent for each diagrammatic step. We did not include the average number of hints requested since only one student used hints for diagrammatic steps. We included grade level and the number of problems solved as covariates in order to keep the models consistent with other models presented earlier. The dependent variables for the three models were the average number of incorrect attempts for each symbolic step, the average time spent for each symbolic step, and the average number of hints requested for each symbolic step. When controlling for these other variables, the average number of incorrect attempts on diagram steps significantly predicted more incorrect attempts on symbolic steps $(\beta=6.17, t(35)=2.50, p=.02)$ and more time spent on symbolic steps $(\beta=25.64, t(35)=2.34, p=.03)$. There was also a significant association between more incorrect attempts on diagrammatic steps and lower hint use on symbolic steps $(\beta=-1.46, t(35)=-2.32, p=.03)$. 


\section{Discussion}

We investigated whether and how anticipatory diagrammatic self-explanation, a novel scaffolding support for learning with visual representations, affects performance and learning for learners with different levels of prior knowledge when integrated into an Intelligent Tutoring System. Our findings indicate that, regardless of their prior knowledge, anticipatory diagrammatic self-explanation helped learners solve symbolic steps faster and ask for fewer hints within the ITS, and supported them in the transition from informal strategies to the formal algebra strategy use for transfer problems with negative numbers on the procedural items in the pretest and posttest. Also, despite the additional diagrammatic steps, which almost doubled the number of steps for each problem, there was no difference, for students with lower prior knowledge, in the number of problems solved between those who received anticipatory diagrammatic self-explanation and those who did not. By contrast, for learners with higher prior knowledge, diagrammatic steps led to fewer problems solved, suggesting that the diagrams introduced additional workload. Still, our results suggest that learners can use inferential activity with tape diagrams to guide their symbolic problem solving. Learners with lower prior knowledge may have used the scaffolding to help with selecting strategic problem-solving steps. For those with higher prior knowledge, although the new representation may have largely captured what they already knew how to do and may not have scaffolded them to solve more problems, it still helped them process symbolic steps faster with fewer hints.

How did anticipatory diagrammatic self-explanation support learning and performance? Our analysis revealed that making more incorrect attempts during anticipatory diagrammatic self-explanation was associated with more time spent and more incorrect attempts made on the symbolic steps, even after controlling for prior knowledge. However, making incorrect diagram selections was also associated with fewer hint requests on the symbolic steps. These results suggest that, although students who make errors on the diagrammatic steps tend to make more errors and spend more time on symbolic steps, anticipatory diagrammatic selfexplanation also serves as a guiding step that learners could use when entering the next symbolic step. Making incorrect diagrammatic self-explanations and receiving feedback on their incorrect attempts may allow learners to reflect on their selection deeply, rather than processing the multiple-choice diagrammatic step shallowly, leading to fewer hint requests made on the symbolic steps. However, we also acknowledge that the observed relation between diagrammatic steps and symbolic steps might be a manifestation of a behavior known as "gaming the system" (Baker et al., 2008). That is, the multiple-choice diagrams with feedback may have invited quick guessing and therefore students may not have fully engaged with diagrams and the ITS.

We did not find any predictors for decreased time spent on the symbolic steps in our model, except the number of problems solved, which was included as a covariate. Further research is necessary, with a larger sample, to explore other potential variables, such as learners' problem-solving performance on the diagrammatic steps for different problem types. Finally, although we had expected to see fewer symbolic problem-solving errors for learners in the Diagram condition, the results did not show a difference in the average number of incorrect attempts made on symbolic steps between the conditions. It may have been that, during symbolic problem-solving, the diagrammatic scaffolding was used as a reference for quicker recovery from errors, but not as a guide for informing how to correctly write the next symbolic step, which has been reported as a challenging translation task for middle-school students (Chu et al., 2017).

We acknowledge several limitations of the study. First, the study focuses on one specific visual representation in one specific domain: tape diagrams for middle-school algebra. The results may or may not generalize to other types of visualizations or other ways of implementing anticipatory diagrammatic self-explanation. Also, a sample size of 84 participants is not large. The additional analysis for the participants in the Diagram condition was performed with an even smaller subset of the data. Therefore, the findings from this study might not correctly reveal causal and predictive relationships. Furthermore, the experiment was conducted remotely during the COVID-19 pandemic, in which the school operated under a special guidance and rules (e.g., socially-distanced layout in the classroom). Given the unique context of the study, it is uncertain whether and to what extent our results generalize to more typical classroom conditions. Lastly, the study was conducted in a private school in the United States. Future studies are necessary to investigate the impact of the intervention with students at other types of schools and in other locations.

\section{Conclusion}

Designing appropriate instructional scaffolding is key to supporting effective and efficient learning with visual representations. Our study demonstrated that anticipatory diagrammatic self-explanation helps learners spend less time and request fewer hints during problem solving. This effect appeared not to vary with learners' prior knowledge. We also found suggestive evidence that anticipatory diagrammatic self-explanation supports the acquisition of the formal algebraic problem-solving strategy. Further, the findings suggested that learners used the diagrams to guide their problem solving with mathematical symbols. Our findings contribute to understanding of effective and efficient instructional scaffolding for learning with visual representations. While prior work had found that diagrams often do not benefit learners with low prior knowledge, the current study suggests that appropriate scaffolding can make benefits of visual representations available to learners who start with lower prior knowledge.

\section{Acknowledgements}

This research was supported by NSF Award \#1760922 and by IES, U.S. Department of Education, through Award 
\#R305B150003 to the UW-Madison. We thank Max Benson, Susan Brunner, Octav Popescu, Jonathan Sewall, Gautam Yadav, and all the students and teachers. This is an author copy. See https://escholarship.org/uc/item/17q6g4db\#main for the original publication.

\section{References}

Ainsworth, S., \& Loizou, T. (2003). The effects of selfexplaining when learning with text or diagrams. Cognitive science, 27(4), 669-681.

Aleven, V. A., \& Koedinger, K. R. (2002). An effective metacognitive strategy: Learning by doing and explaining with a computer-based cognitive tutor. Cognitive Science, 26(2), 147-179.

Baker, R., Walonoski, J., Heffernan, N., Roll, I., Corbett, A., \& Koedinger, K. (2008). Why students engage in "gaming the system" behavior in interactive learning environments. Journal of Interactive Learning Research, 19(2), 185-224.

Barbieri, C. A., Miller-Cotto, D., \& Booth, J. L. (2019). Lessening the load of misconceptions: Design-based principles for algebra learning. Journal of the Learning Sciences, 28(3), 381-417.

Bartel, A. N., Silla, E. M., Vest, N. A., Nagashima, T., Aleven, V., \& Alibali, M. W. (2021). Reasoning about equations with tape diagrams: insights from math teachers and college students. In Proceedings of the Annual Meeting of the International Society of the Learning Sciences. Bochum, Germany.

Bisra, K., Liu, Q., Nesbit, J. C., Salimi, F., \& Winne, P. H. (2018). Inducing self-explanation: A meta-analysis. Educational Psychology Review, 30, 703-725.

Booth, J. L., \& Koedinger, K. R. (2012). Are diagrams always helpful tools? Developmental and individual differences in the effect of presentation format on student problem solving. British Journal of Educational Psychology, 82(3), 492-511.

Butcher, K. R., \& Aleven, V. (2013). Using student interactions to foster rule-diagram mapping during problem solving in an intelligent tutoring system. Journal of Educational Psychology, 105(4), 988.

Chi, M. T., Bassok, M., Lewis, M. W., Reimann, P., \& Glaser, R. (1989). Self-explanations: How students study and use examples in learning to solve problems. Cognitive Science, 13(2), 145-182.

Chu, J., Rittle-Johnson, B., \& Fyfe, E. R. (2017). Diagrams benefit symbolic problem-solving. British Journal of Educational Psychology, 87(2), 273-287.

Crooks, N. M., \& Alibali, M. W. (2014). Defining and measuring conceptual knowledge in mathematics. Developmental Review, 34(4), 344-377.

Davenport, J., Yaron, D., Klahr, D., \& Koedinger, K. (2008). When do diagrams enhance learning? A framework for designing relevant representations. In Proceedings of the International Conference of the Learning Sciences (pp. 191-198).

Delen, E., Liew, J., \& Willson, V. (2014). Effects of interactivity and instructional scaffolding on learning:
Self-regulation in online video-based environments. Computers \& Education, 78, 312-320.

Long, Y., \& Aleven, V. (2013). Supporting students' selfregulated learning with an open learner model in a linear equation tutor. In International Conference on Artificial Intelligence in Education (pp. 219-228). Springer, Berlin, Heidelberg.

Long, Y., Aleven, V. (2014). Gamification of joint student/system control over problem selection in a linear equation tutor. In S. Trausan-Matu, K. E. Boyer, M. Crosby, \& K. Panourgia (Eds.), In Proceedings of the 12th International Conference on Intelligent Tutoring Systems (pp. 378-387). New York: Springer.

Magner, U. I., Schwonke, R., Aleven, V., Popescu, O., \& Renkl, A. (2014). Triggering situational interest by decorative illustrations both fosters and hinders learning in computer-based learning environments. Learning and Instruction, 29, 141-152.

Murata, A. (2008). Mathematics teaching and learning as a mediating process: The case of tape diagrams. Mathematical Thinking and Learning, 10(4), 374-406.

Nagashima, T., Bartel, A. N., Silla, E. M., Vest, N. A., Alibali, M. W., \& Aleven, V. (2020). Enhancing conceptual knowledge in early algebra through scaffolding diagrammatic self-explanation. In M. Gresalfi \& I. S. Horn (Eds.), Proceedings of International Conference of the Learning Sciences (pp. 35-43). Nashville, TN: International Society of the Learning Sciences.

Nagashima, T., Bartel, A. N., Yadav, G., Tseng, S., Vest, N. A., Silla, E. M., Alibali, M. W., \& Aleven, V. (2021). Using anticipatory diagrammatic self-explanation to support learning and performance in early algebra. In Proceedings of the Annual Meeting of the International Society of the Learning Sciences. Bochum, Germany.

Nagashima, T., Yang. K., Bartel, A. N., Silla, E. M., Vest, N. A., Alibali, M. W., \& Aleven, V. (2020). Pedagogical Affordance Analysis: Leveraging teachers' pedagogical knowledge for eliciting pedagogical affordances and constraints of instructional tools. In M. Gresalfi \& I. S. Horn (Eds.), Proceedings of International Conference of the Learning Sciences (pp. 1561-1564). Nashville, TN: International Society of the Learning Sciences.

Rau, M. A. (2017). Conditions for the effectiveness of multiple visual representations in enhancing STEM learning. Educational Psychology Review, 29(4), 717-761.

Rau, M. A., Aleven, V., \& Rummel, N. (2015). Successful learning with multiple graphical representations and self-explanation prompts. Journal of Educational Psychology, 107(1), 30.

Renkl, A. (1997). Learning from worked-out examples: A study on individual differences. Cognitive Science, 21(1), $1-29$.

Schwartz, D. L., Chase, C. C., Oppezzo, M. A., \& Chin, D. B. (2011). Practicing versus inventing with contrasting cases: The effects of telling first on learning and transfer. Journal of Educational Psychology, 103(4), 759. 